\title{
Time to take CT screening to the next level?
}

\author{
Hans-Ulrich Kauczor ${ }^{1,2,3}$, Claus Peter Heussel ${ }^{1,2,3}$ and \\ Oyunbileg von Stackelberg ${ }^{1,2,3}$
}

Affiliations: ${ }^{1}$ Dept of Diagnostic and Interventional Radiology, University Medical Center Heidelberg, Heidelberg, Germany. ${ }^{2}$ Translational Lung Research Center (TLRC) Heidelberg, Member of the German Center for Lung Research (DZL), Heidelberg, Germany. ${ }^{3}$ Dept of Diagnostic and Interventional Radiology with Nuclear Medicine, Thoraxklinik, University Medical Center Heidelberg, Heidelberg, Germany.

Correspondence: Hans-Ulrich Kauczor, Dept of Diagnostic and Interventional Radiology, University Medical Center Heidelberg, Im Neuenheimer Feld 110, 69120 Heidelberg, Germany.

E-mail: Hans-Ulrich.Kauczorlamed.uni-heidelberg.de

@ERSpublications

Transforming lung cancer screening into a comprehensive programme to detect and treat smokingrelated disease early http://ow.ly/b8L5309hUri

Cite this article as: Kauczor H-U, Heussel CP, von Stackelberg O. Time to take CT screening to the next level?. Eur Respir J 2017; 49: 1700064 [https://doi.org/10.1183/13993003.00064-2017].

The paper by Pompe et al. [1] in this edition of the European Respiratory Journal investigated subjects from the National Lung Cancer Screening Trial (NLST), whose cause of death was recorded as being a respiratory illness other than lung cancer. The patients' computed tomography (CT) scans were retrospectively reviewed and compared with the NLST participants of the CT arm who were still alive. Not surprisingly, significantly more lung diseases were detected in those who died from respiratory disease than in the matched control group.

We are all aware that the NLST has demonstrated a significant reduction in lung cancer mortality [2]. An additional finding of NLST showed that all-cause mortality was also significantly lower in participants screened with CT. When deaths from lung cancer were excluded from the comparison, the reduction in overall mortality with the use of low-dose CT dropped to $3.2 \%$ and was not significant anymore [2].

For their retrospective case-control study, the authors hypothesised that the pulmonary abnormalities in accordance with respiratory deaths should already be visible on the CT scans and that the NLST data provided a unique opportunity to investigate the importance of these CT diagnoses for survival [1]. Thus, the CT scans were visually assessed for the prevalence of emphysema (sub-classified as mild, moderate or severe/confluent and advanced destructive emphysema) [3], airway wall thickening and fibrotic lung disease. POMPe et al. [1] identified among the NLST participants 167 people who died from a respiratory disease other than lung cancer, most of them from chronic obstructive pulmonary disease (COPD). The authors double-checked the cause of death to put their results on stable ground. As might have been expected, these participants had significantly more pulmonary disease visible on the CT scan (severe emphysema, airway abnormalities and fibrotic lung disease) than the surviving matched-pair controls.

For their analysis, the authors relied on a very simple, visual, subjective approach. They had two radiologists with different levels of expertise reading the scans. They took simple binary decisions: presence or absence of severe emphysema, airway abnormalities or fibrosis. Obviously, this approach was very fast, and it took them less than a minute per scan. The results are sufficiently robust, with a high inter-reader agreement (weighted kappa 0.7-0.91). It is important to note that these results were achieved

Received: Jan 122017 | Accepted after revision: Jan 192017

Conflict of interest: Disclosures can be found alongside this article at erj.ersjournals.com

Copyright @ERS 2017 
using low-dose CT scans, which were not intended to serve for quantitative analysis. The disease classification could be made with appropriate confidence despite higher image noise compared with routine "diagnostic" CT scans.

However, the good results and the prompt visual assessment are just an intermediate step on the way to software-based pre-reads and automated reproducible disease quantification, which should be implemented in future imaging-based screening scenarios. The change from a visual binary categorisation to contiguous quantitative data will make the output much more valuable [4]. Dedicated software tools will also be capable of performing more complex analysis, such as feature extraction (radiomics) of smoking-related diseases and co-morbidities (such as emphysema, bronchial-wall thickening, fibrosis, arteriosclerosis, sarcopenia and osteopenia), and the results will be fully eligible for "big data" analysis tools [5]. Such an approach will generate deeper phenotypic insights into unexpected associations.

At first glance, the results of this retrospective analysis seem rather trivial - at least for a radiologist assessing chest CTs every day. Smokers who die from pulmonary disease have more abnormalities visible on CT scan, and a higher number of abnormalities seen on the scan carries a higher risk for dying from respiratory disease. This clearly emphasises that all CT-detectable abnormalities should be included when screening a high-risk population, as severe COPD and fibrosis are associated with very limited life expectancy even without development of synchronous lung cancer [6-8].

However, the results of this study go beyond this simple assumption and confirm the value of the high sensitivity of CT for the detection of respiratory diseases, especially COPD. CT was also shown to have a clear impact on the outcome of the participants or patients, which is a core requirement in value-based medicine, which is also applicable to radiology. If done properly, either sticking to the algorithms of the NLST or even using matured algorithms, CT screening of lung cancer will reduce lung cancer mortality in high-risk smokers. Because of the evidence generated by the NLST, lung cancer screening with annual low-dose CT has been introduced in the US in adults aged 55-80 years who have a 30 pack-year smoking history and currently smoke or have quit within the past 15 years [9], with cancer incidence in lung cancer screening dropping dramatically after the first round [10]. Longer screening intervals result in lower cumulative radiation dose, but intervals as long as 2.5 years are not recommended because there is a higher interval cancer rate and a higher proportion of advanced-stage disease [11].

Recommendations for the potential implementation in Europe were published jointly by the European Respiratory Society and the European Society of Radiology in 2015 [12, 13]. Several calculations and simulations demonstrate that CT screening for lung cancer can be cost-effective $[14,15]$. Surprisingly, a comprehensive approach towards the early detection of the "Big Three" killers associated with cigarette smoking (lung cancer, COPD and cardiovascular disease) is still missing, despite the fact that all of them are more or less visible on a single nongated, nonenhanced, low-dose CT scan. Pompe et al. [1] nicely demonstrated the outcome-based value of the CT diagnosis of emphysema, airway remodelling and COPD within lung cancer screening programmes. They confirmed and enriched the results from other studies, showing the value of reporting abnormalities related to "COPD", especially emphysema and other interstitial abnormalities. It is known that patients with emphysema have a higher risk of developing lung cancer, and the greater the emphysema, the higher the risk [16].

The value of diagnosing fibrotic lung disease in smokers has been evaluated only in a single site of NLST, and showed a prevalence of almost $10 \%$ and a progression of $37 \%$ at 2 -year follow-up [17]. In addition, the combination of COPD and fibrosis as a smoking-related lung disease has received increasing interest recently, and a new term, "combined pulmonary fibrosis and emphysema" (CPFE), has been coined [18, 19].

To take CT screening of the lung to the next level, the risk for cardiovascular events has to be assessed as well. Image-wise, smoking-associated cardiovascular disease is best represented by the extent of vascular calcifications. The nongated, nonenhanced, low-dose scans obtained in lung cancer screening cohorts are well suited to diagnose cardiovascular calcifications [20], which are a well-established risk factor for cardiovascular events and death [21-23]. The findings have been grouped into coronary artery calcification (CAC) and extracoronary calcification (ECC), and can be established accurately by visual and/or quantitative analysis. There is a strong association between ECC load and established cardiovascular risk factors such as smoking history, hypertension, diabetes and hypercholesterolaemia [21]. Using a simple visual assessment of CAC on a low-dose CT scan, a risk assessment of coronary heart disease death and all-cause mortality can be generated, which is comparable to the Agatston score and strongly associated with outcome [24]. But again, a visual binary assessment will do as a first step, while a software-based assessment will provide a more extensive basis for further risk calculations.

There are even debates about extending the scanning field of low-dose chest CT to the neck. This would allow for assessment of vascular calcifications as well as the lipid core of the plaques in the carotid vessel 
in the same examination. The impact of these findings on the risk profile of smokers still has to be determined, however [25].

In order to obtain comprehensive data on the "Big Three" from a single low-dose screening CT, a dedicated software tool is required, which will depict and measure lung nodules, low-attenuation areas (emphysema), airway wall and lumen, "fibrosis", and coronary and extracoronary arterial calcification load. Furthermore, such a software tool should be capable both of obtaining radiomics data by feature analysis (e.g. malignancy potential of a nodule, lipid core of a plaque), and of measuring changes in the subsequent screening rounds or follow-ups. The image-based "phenotypic" biomarkers of risk should be complemented by minimally invasive biomarkers from blood, serum, plasma ("liquid biopsy") $[26,27]$ or exhaled breath $[28,29]$ in the future, either to increase pre-test probability or to reduce the number of false-positive results.

Such comprehensive results of a screening programme must not be used as an excuse for patients to continue smoking but rather to increase the individual success rate of smoking cessation. Confronting the participants with convincing visual findings from their scans will increase the awareness of the vulnerability of their own body and increase the motivation to stop smoking, e.g. evidence of nodules increases the success of smoking cessation from $5 \%$ to $40 \%$ [30].

In conclusion, it is time to reshape our conception of lung cancer screening and take CT screening to the next more holistic level. We have to fight the reduced life expectancy of heavy (i.e. high-risk) smokers, which is about 10-12 years lower than in never-smokers. To be successful, two strategies have to be merged: (1) to stop smoking and (2) to detect and treat survival-limiting, smoking-related disease early. This should be done by a comprehensive screening programme for heavy smokers, offering a smoking cessation programme together with a risk-model based CT screen and biomarker analysis of the "Big Three" smoking-related killer diseases: lung cancer, COPD and cardiovascular disease.

\section{References}

1 Pompe E de Jong PA, Lynch DA, et al. Computed tomographic findings in subjects who died from respiratory disease in the National Lung Screening Trial. Eur Respir J 2017; 49: 1601814.

2 Aberle DR, Adams AM, Berg CD, et al. Reduced lung-cancer mortality with low-dose computed tomographic screening. N Engl J Med 2011; 365: 395-409.

3 Lynch DA, Austin JH, Hogg JC, et al. CT-definable subtypes of chronic obstructive pulmonary disease: a statement of the Fleischner Society. Radiology 2015; 277: 192-205.

4 Ginsburg SB, Zhao J, Humphries S, et al. Texture-based quantification of centrilobular emphysema and centrilobular nodularity in longitudinal ct scans of current and former smokers. Acad Radiol 2016; 23: 1349-1358.

5 Hawkins S, Wang H, Liu Y, et al. Predicting malignant nodules from screening CT scans. J Thorac Oncol 2016; 11: $2120-2128$.

6 Jairam PM, van der Graaf Y, Lammers JW, et al. Incidental findings on chest CT imaging are associated with increased COPD exacerbations and mortality. Thorax 2015; 70: 725-731.

7 Le Jeune I, Gribbin J, West J, et al. The incidence of cancer in patients with idiopathic pulmonary fibrosis and sarcoidosis in the UK. Respir Med 2007; 101: 2534-2540.

8 Ozawa Y, Suda T, Naito T, et al. Cumulative incidence of and predictive factors for lung cancer in IPF. Respirology 2009; 14: 723-728.

9 US Preventive Services Task Force. Final recommendation statement: lung cancer: screening. www. uspreventiveservicestaskforce.org/Page/Document/RecommendationStatementFinal/lung-cancer-screening Date last accessed: January 2017; date last updated: December 2013.

10 Becker N, Delorme S. Voraussetzungen für ein erfolgreiches Lungenkrebsscreeningprogramm. [Prerequisites for a successful lung cancer screening programme]. Der Radiologe 2016; 56: 786-792.

11 Yousaf-Khan U, van der Aalst C, de Jong PA, et al. Final screening round of the NELSON lung cancer screening trial: the effect of a 2.5-year screening interval. Thorax 2017; 72: 48-56.

12 Kauczor HU, Bonomo L, Gaga M, et al. ESR/ERS white paper on lung cancer screening. Eur Respir J 2015; 46: 28-39.

13 Kauczor HU, Bonomo L, Gaga M, et al. ESR/ERS white paper on lung cancer screening. Eur Radiol 2015; 25: 2519-2531.

14 Black WC, Gareen IF, Soneji SS, et al. Cost-effectiveness of CT screening in the National Lung Screening Trial. N Engl J Med 2014; 371: 1793-1802.

15 Field JK, Duffy SW, Baldwin DR, et al. UK Lung Cancer RCT Pilot Screening Trial: baseline findings from the screening arm provide evidence for the potential implementation of lung cancer screening. Thorax 2016; 71: $161-170$.

16 Wille MM, Thomsen LH, Petersen J, et al. Visual assessment of early emphysema and interstitial abnormalities on CT is useful in lung cancer risk analysis. Eur Radiol 2016; 26: 487-494.

17 Jin GY, Lynch D, Chawla A, et al. Interstitial lung abnormalities in a CT lung cancer screening population: prevalence and progression rate. Radiology 2013; 268: 563-571.

18 Chae KJ, Jin GY, Han YM, et al. Prevalence and progression of combined pulmonary fibrosis and emphysema in asymptomatic smokers: A case-control study. Eur Radiol 2015; 25: 2326-2334.

19 Choi SH, Lee HY, Lee KS, et al. The value of CT for disease detection and prognosis determination in combined pulmonary fibrosis and emphysema (CPFE). PloS One 2014; 9: e107476.

20 Heussel CP, Voigtlaender T, Kauczor H, et al. Detection of coronary artery calcifications predicting coronary heart disease: comparison of fluoroscopy and spiral CT. Eur Radiol 1998; 8: 1016-1024. 
21 Dirrichs T, Penzkofer T, Reinartz SD, et al. Extracoronary thoracic and coronary artery calcifications on chest ct for lung cancer screening: association with established cardiovascular risk factors - The "CT-Risk" Trial. Acad Radiol 2015; 22: 880-889.

22 Jacobs PC, Prokop M, van der Graaf Y, et al. Comparing coronary artery calcium and thoracic aorta calcium for prediction of all-cause mortality and cardiovascular events on low-dose non-gated computed tomography in a high-risk population of heavy smokers. Atherosclerosis 2010; 209: 455-462.

23 Williams MC, Murchison JT, Edwards LD, et al. Coronary artery calcification is increased in patients with COPD and associated with increased morbidity and mortality. Thorax 2014; 69: 718-723.

24 Chiles C, Duan F, Gladish GW, et al. Association of coronary artery calcification and mortality in the National Lung Screening Trial: a comparison of three scoring methods. Radiology 2015; 276: 82-90.

25 van den Bouwhuijsen QJ, Bos D, Ikram MA, et al. Coexistence of calcification, intraplaque hemorrhage and lipid core within the asymptomatic atherosclerotic carotid plaque: The Rotterdam Study. Cerebrovasc Dis 2015; 39: 319-324.

26 Hofman P. Liquid biopsy for early detection of lung cancer. Curr Opin Oncol 2017; 29: 73-78.

27 Leidinger P, Keller A, Borries A, et al. Specific peripheral miRNA profiles for distinguishing lung cancer from COPD. Lung Cancer 2011; 74: 41-47.

28 Nakhleh MK, Amal H, Jeries R, et al. Diagnosis and classification of 17 diseases from 1404 subjects via pattern analysis of exhaled molecules. ACS Nano 2016; 11: 112-125.

29 Peled N, Hakim M, Bunn PA Jr, et al. Non-invasive breath analysis of pulmonary nodules. J Thorac Oncol 2012; 7: 1528-1533.

30 Young RP, Hopkins RJ, Smith M, et al. Smoking cessation: the potential role of risk assessment tools as motivational triggers. Postgrad Med J 2010; 86: 26-33. 